\title{
SUSPENSION SPLITTINGS AND SELF-MAPS OF FLAG MANIFOLDS
}

\author{
SHIZUO KAJI AND STEPHEN THERIAULT
}

\begin{abstract}
Авstract. If $G$ is a compact connected Lie group and $T$ is a maximal torus, we give a wedge decomposition of $\Sigma G / T$ by identifying families of idempotents in cohomology. This is used to give new information on the self-maps of $G / T$.
\end{abstract}

\section{INTRODUCTION}

Let $G$ be a compact connected Lie group and let $T$ be a maximal torus. There has been considerable interest in trying to determine the homotopy classes of the self-maps of the quotient space $G / T$. One method commonly adopted in $[6,9,19,26]$ is to study the image of the map

$$
r:[G / T, G / T] \longrightarrow \operatorname{Hom}_{\text {alg }}\left(H^{*}(G / T), H^{*}(G / T)\right) .
$$

We show that if $G$ is simply-connected there is a bijection

$$
[G / T, G / T] \cong[G / T, G] \times \operatorname{Im}(r),
$$

where $r$ sends a self-map to the ring homomorphism it induces in cohomology. This was earlier claimed to hold in more generality in [27], but there seems to be gaps. With (1) in hand, we consider the other factor, $[G / T, G]$, and develop an approach to understanding it.

Since $G$ is a group it has a classifying space $B G$. This implies that there is a group isomorphism $[G / T, G] \cong[\Sigma G / T, B G]$. The idea is to decompose $\Sigma G / T$ into a wedge of smaller spaces which simplify the calculations. The decompositions are obtained by identifying certain idempotents in cohomology. These are $p$-local decompositions, where $p$ is a prime. Two families of idempotents are considered, one coming from Adams operations on the classifying spaces of $T$ and $G$, the other coming from the action of the Weyl group on $G / T$. These are also compatible in the sense that they can be merged to form a larger set of idempotents, giving a finer decomposition of the space.

These suspension splittings also fit into a larger framework that considers stable decompositions of homogeneous spaces. The classic example of this is Miller's stable splitting of Stiefel manifolds [16], which inspired a great many variants and refinements (e.g., [12, 18, 24, 25]). In those cases, the stable feature is prominent in the sense that multiple suspensions are usually needed to realize the decomposition, whereas in our case the decomposition occurs after a single suspension.

To demonstrate the methods we give explicit decompositions of $S U(3) / T, S U(4) / T, S p(2) / T$ and $G_{2} / T$, and go on to calculate $[G / T, G]$ in each case (modulo 2-primary information in the $S U(4)$ and $G_{2}$ cases).

\section{The COHOMOLOGY of $G / T$}

Let $W=N(T) / T$ be the Weyl group of $G$, which is generated by the simple reflections $s_{1}, \ldots, s_{r}$, where $r=\operatorname{rank}(T)$.

Date: May 13, 2018.

2010 Mathematics Subject Classification. Primary 55P40, 55S37, Secondary 57 T15.

Key words and phrases. flag manifold, self-map, stable splitting.

This work was supported by KAKENHI, Grant-in-Aid for Scientific Research (C) 18 K03304. 
Definition 2.1. For an element of $w \in W$, the length $l(w)$ of $w$ is the least integer such that $w$ can be written as a product of $l(w)$ simple reflections. So an element $w$ of length $n$ can be written as $w=s_{i_{1}} s_{i_{2}} \cdots s_{i_{n}}$ for some sequence of simple reflections. We often abbreviate this as $w=s_{i_{1} i_{2} \cdots i_{n}}$.

The following theorem proved by [8] describes $H^{*}(G / T ; \mathbb{Z})$ as a free $\mathbb{Z}$-module.

Theorem 2.2 (Bruhat decomposition). There is a cell decomposition

$$
G / T=\bigcup_{w \in W} \sigma_{w},
$$

where $\sigma_{w}$ are open cells with $\operatorname{dim}\left(\sigma_{w}\right)=2 l(w)$. Moreover, the closure is $\overline{\sigma_{w}}=\bigcup_{v \leq w} \sigma_{v}$, where the order on $W$ is given by the strong Bruhat order, that is, $v \leq w$ iff a reduced word for $w$ contains one of $v$ as a sub-word. Consequently, $H^{*}(G / T ; \mathbb{Z})$ is torsion free of rank $|W|$ and its basis is given by the Schubert classes:

$$
H^{*}(G / T ; \mathbb{Z}) \cong H^{\text {even }}(G / T ; \mathbb{Z}) \simeq \mathbb{Z}\left\langle\sigma_{w}\right\rangle_{w \in W}
$$

Borel [3] gives another description of $H^{*}(G / T)$ as the quotient of a polynomial ring.

Theorem 2.3 (Coinvariant description). Let $R$ be a ring in which the torsion primes [4] of $G$ are inverted. Then

$$
H^{*}(G / T ; R)=H^{*}(B T ; R) / I
$$

where $I$ is the ideal generated by the Weyl group invariants of positive degree.

Example 2.4.

$$
H^{*}\left(\operatorname{SU}(n) / T^{n-1} ; \mathbb{Z}\right)=\mathbb{Z}\left[x_{1}, x_{2}, \ldots, x_{n}\right] /\left(e_{1}, e_{2}, \ldots, e_{n}\right),
$$

where $e_{i}$ is the $i$-th elementary symmetric function on $x_{1}, \ldots, x_{n}$. A choice of a polynomial representative of $\sigma_{w}$ in this presentation is given by the classical Schubert polynomial [13].

3. A splitting of $[G / T, G / T]$

The group homomorphism $T \longrightarrow G$ classifies, giving a homotopy fibration sequence

$$
T \longrightarrow G \stackrel{q}{\longrightarrow} G / T \stackrel{j}{\longrightarrow} B T \longrightarrow B G
$$

which defines the map $j$. In particular, for a simply-connected space $X$ we obtain an exact sequence

$$
[X, G] \stackrel{q_{*}}{\longrightarrow}[X, G / T] \stackrel{j_{*}}{\longrightarrow}[X . B T]
$$

where $q_{*}$ is injective. Consider the map

$$
r:[X, G / T] \longrightarrow \operatorname{Hom}_{\mathrm{alg}}\left(H^{*}(G / T ; \mathbb{Z}), H^{*}(X ; \mathbb{Z})\right)
$$

defined by sending a map $X \longrightarrow G / T$ to the algebra homomorphism it induces in cohomology. Similarly, there is a map

$$
[X, B T] \longrightarrow \operatorname{Hom}_{\mathrm{alg}}\left(H^{*}(B T ; \mathbb{Z}), H^{*}(X ; \mathbb{Z})\right)
$$

which is an isomorphism since both sides are canonically isomorphic to $\bigoplus_{1 \leq i \leq r} H^{2}(X ; \mathbb{Z})$. We obtain a commutative diagram

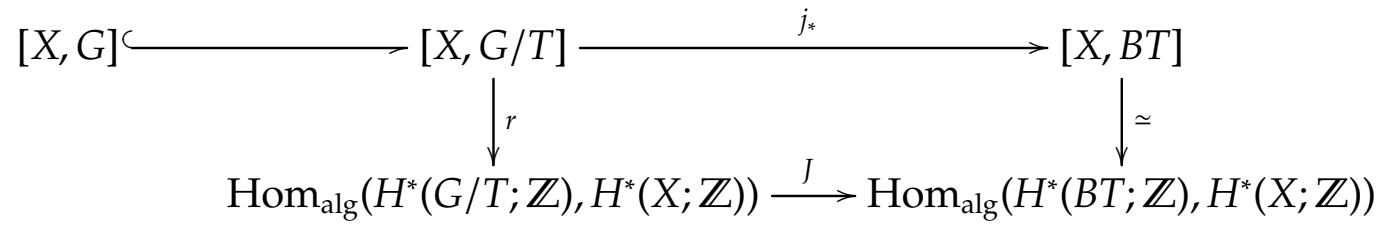

where $J\left(f^{*}\right)=f^{*} \circ j^{*}$. We have the holonomy action $[X, G / T] \times[X, G] \rightarrow[X, G / T]$. By the Puppe sequence [14, Lemma 1.4.7], the action is free and moreover, for $f_{1}, f_{2} \in[X, G / T]$ we have 
$j_{*}\left(f_{1}\right)=j_{*}\left(f_{2}\right)$ if and only if $f_{1}$ and $f_{2}$ are in the same orbit of the action of $[X, G]$. Therefore, we have the following non-canonical identification

$$
[X, G / T] \cong[X, G] \times \operatorname{Im}\left(j_{*}\right) \text {. }
$$

Proposition 3.1. If $X$ is simply-connected and $H^{*}(X ; \mathbb{Z})$ is torsion-free then the map $J$ is a monomorphism.

Proof. Since $H^{*}(G / T ; \mathbb{Z}), H^{*}(X ; \mathbb{Z})$, and $H^{*}(B T ; \mathbb{Z})$ are torsion free, the vertical maps (rationalizations) in the following commutative diagram are injective

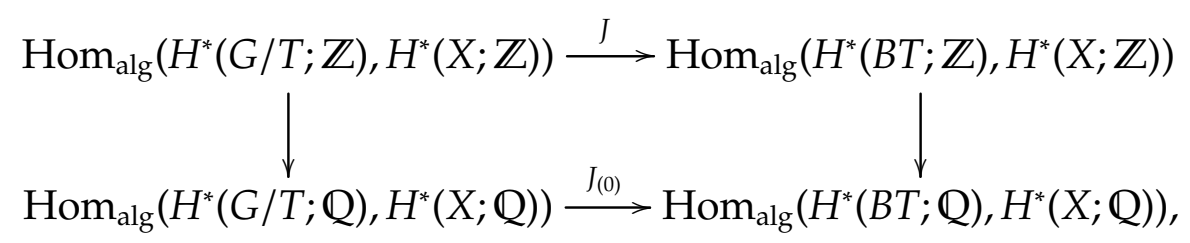

where $J_{(0)}$ is the rationalization of $J$. Since $H^{*}(G / T ; \mathbb{Q})$ is generated by the degree two elements and $H^{2}(G / T ; \mathbb{Q}) \cong H^{2}(B T ; \mathbb{Q})$, we see that $J_{(0)}$ is injective. The commutativity of the diagram then implies that $J$ is also injective.

Remark 3.2. Zhao [27, Lemma 1] claims that $J$ is injective without the torsion-free hypothesis. However, this seems unlikely. Observe that $H^{*}(G / T ; \mathbb{Z})$ is torsion-free and $H^{*}(G / T ; \mathbb{Q})$ is generated by degree two elements. But $H^{*}(G / T ; \mathbb{Z})$ is NOT generated by degree two elements in general; for example in the case of $H^{*}\left(G_{2} / T ; \mathbb{Z}\right)$ described in $\S 5.3$, even when an induced map $f^{*}$ for $f \in[X, G / T]$ is trivial on $H^{2}\left(G_{2} / T ; \mathbb{Z}\right) \simeq \mathbb{Z}\left[x_{1}, x_{2}, x_{3}\right] /\left(e_{1}\right), f^{*}(\gamma)$ can be a non-trivial torsion element in $H^{*}(X ; \mathbb{Z})$.

Corollary 3.3. If $X$ is simply-connected and $H^{*}(X)$ is torsion-free then there is an isomorphism

$$
[X, G / T] \cong[X, G] \times \operatorname{Im}(r) .
$$

Proof. By Proposition 3.1, the map J in (2) is a monomorphism. The square in (2) therefore implies that $\operatorname{Im}\left(j_{*}\right) \cong \operatorname{Im}(r)$. Now substitute this isomorphism into (3).

By Theorem 2.2, $H^{*}(G / T)$ is torsion-free. So Proposition 3.1 immediately implies the following.

Corollary 3.4. Let $G$ be a compact simply-connected Lie group. Then there is an isomorphism

$$
[G / T, G / T] \cong[G / T, G] \times \operatorname{Im}(r) .
$$

Note that $G / T \simeq \hat{G} / \hat{T}$, where $\hat{G}$ is the universal cover of $G$ and $\hat{T}$ is the maximal torus of $\hat{G}$. Hence, we can always take $G$ to be simply-connected.

\section{IDEMPOTENTS FOR $H^{*}(G / T)$}

Now we start to focus on $[G / T, G]$, which by Corollary 3.4 is a factor of $[G / T, G / T]$. In this section we construct two families of compatible idempotents for $H^{*}(G / T)$ and use them to produce wedge decompositions of $\Sigma G / T$. This begins with a general lemma (c.f. [20, §2]).

Definition 4.1. Let $R$ be a ring. A finite collection $p_{1}, p_{2}, \ldots, p_{n}$ of self-maps of a connected space $X$ is called a set of mutually orthogonal idempotents of $H^{*}(X ; R)$ if:

(i) $p_{i}^{*} \circ p_{i}^{*}=p_{i}^{*}$ for $1 \leq i \leq n$;

(ii) $p_{i}^{*} \circ p_{j}^{*}=0$ for all $1 \leq i, j \leq n$ with $i \neq j$; and

(iii) $p_{1}^{*}+\cdots+p_{n}^{*}=1$.

Given a self-map $f: X \rightarrow X$, let $\operatorname{Tel}(f)$ be the telescope of $f$ and let $t: X \rightarrow \operatorname{Tel}(f)$ be the map to the telescope. Since $t \circ f \simeq t$, the $\operatorname{map} H^{*}(\operatorname{Tel}(f) ; R) \stackrel{t^{*}}{\longrightarrow} H^{*}(X ; R)$ induces the inclusion of $\operatorname{Im}\left(f^{*}\right)$. 
Lemma 4.2. Let $X$ be a simply-connected finite co- $H$-space. Let $p_{1}, \ldots, p_{n}$ be a set of mutually orthogonal idempotents on $H^{*}(X ; \mathbb{Z} / p \mathbb{Z})$. Then there is a $p$-local homotopy equivalence

$$
X \simeq \bigvee_{i=1}^{n} \operatorname{Tel}\left(p_{i}\right)
$$

Proof. Since $X \stackrel{p_{i}}{\longrightarrow} \operatorname{Tel}\left(p_{i}\right)$ induces the inclusion of $\operatorname{Im}\left(p_{i}^{*}\right)$, the sum of the maps $p_{i}$ defines a map $\psi: X \rightarrow \bigvee_{i=1}^{n} \operatorname{Tel}\left(p_{i}\right)$ which induces an isomorphism in mod- $p$ cohomology. Since $X$ is simply-connected and of finite type, this implies that $\psi$ is a $p$-local homotopy equivalence by [10, Chapter II, Theorem 1.14].

We identify two families of self maps of $\Sigma G / T$ that can be used to produce idempotents on $H^{*}(\Sigma G / T ; \mathbb{Z} / p \mathbb{Z})$. Note that the co- $H$ structure on $\Sigma G / T$ induces a group structure on $[\Sigma G / T, \Sigma G / T]$.

4.1. Unstable Adams operations. We follow the argument in [25]. For $l \in \mathbb{Z}$ prime to $|W|$, there is a commutative diagram

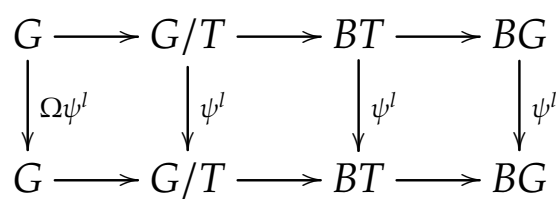

where $\left(\psi^{l}\right)^{*}: H^{2 i}(X) \rightarrow H^{2 i}(X)$ is multiplication by $l^{i}$. by

For an odd prime $p$, choose $l \in \mathbb{Z}$ which is primitive in $\mathbb{F}_{p}^{\times}$and define a self-map of $\Sigma G / T$

$$
\phi_{i}=\Sigma \psi^{l}-l^{i} \text { and } \varphi_{k}^{\prime}=\prod_{1 \leq i \leq n, i \neq k \bmod p-1} \phi_{i}
$$

where $n=\max (p-1, \operatorname{dim}(G / T) / 2)$ and $l^{i}: \Sigma G / T \rightarrow \Sigma G / T$ is $l^{i}$ times the identity map. Note that $\varphi_{i}^{\prime}$ is trivial on $H^{2 j+1}(\Sigma G / T ; \mathbb{Z} / p \mathbb{Z})$ iff $j=i \bmod p-1$. So by normalizing up to unit, we obtain a set of mutually orthogonal idempotents $\varphi_{1}, \ldots, \varphi_{n}$ on $H^{*}(\Sigma G / T ; \mathbb{Z} / p \mathbb{Z})$, where $\varphi_{i}=u_{i} \varphi_{i}^{\prime}$ for some unit $u_{i} \in \mathbb{Z} / p \mathbb{Z}$. Therefore, by Lemma 4.2 there is a $p$-local homotopy equivalence

$$
\Sigma G / T \simeq \bigvee \operatorname{Tel}\left(\varphi_{i}\right)
$$

where $\tilde{H}^{2 i+1}\left(\operatorname{Tel}\left(\varphi_{k}\right) ; \mathbb{Z} / p \mathbb{Z}\right) \cong \begin{cases}\tilde{H}^{2 i}(G / T ; \mathbb{Z} / p \mathbb{Z}) & \text { if } i=k \bmod p-1 \\ 0 & \text { if } i \neq k \bmod p-1\end{cases}$

4.2. The Weyl group action. The flag manifold $G / T$ is equipped with a right Weyl group action:

$$
g T \mapsto g w T
$$

for $w \in W=N(T) / T$. Thus, given any $w \in W$ we obtain a self-map $w: G / T \longrightarrow G / T$. In particular, each simple reflection $s_{i}$ induces a self-map $s_{i}: G / T \longrightarrow G / T$.

By [2], the $W$-action on Schubert classes is given by

$$
s_{i} \sigma_{w}= \begin{cases}\sigma_{w} & \text { if } l\left(w s_{i}\right)=l(w)+1 \\ -\sigma_{w}-\sum_{l\left(w s_{i} s_{\beta}\right)=l(w)} \frac{2\left(\beta, \alpha_{i}\right)}{(\beta, \beta)} \sigma_{w s_{\beta}} & \text { if } l\left(w s_{i}\right)=l(w)-1 .\end{cases}
$$

In the coinvariant description (Theorem 2.3), the $W$-action is simply induced by the ordinary one on $H^{*}(B T ; R)$.

Using the co- $H$-structure on $\Sigma G / T$ to add maps, to each element $v$ in the group ring $\mathbb{Z}[W]$ there associated a self-map $v: \Sigma G / T \longrightarrow \Sigma G / T$. Thus if we find a set of mutually orthogonal idempotents in the group ring we can find an induced set of mutually orthogonal 
idempotents in $H^{*}(\Sigma G / T ; \mathbb{Z})$. The same argument works if we replace $\mathbb{Z}$-coefficients with $\mathbb{Z} / p \mathbb{Z}$ or $\mathbb{Q}$-coefficients and consider the corresponding localization of the space.

It is well-known that $H^{*}(G / T ; \mathbb{Q})$ is the regular representation of $W$ and decomposes into irreducible representations. However, constructing the corresponding set of mutually orthogonal idempotents even in $\mathbb{Q}[W]$ is non-trivial [1]. For our purpose, we aim to construct mutually orthogonal idempotents in $\mathbb{Z} / p \mathbb{Z}[W]$, and in the examples in Section 5, the identification of the mutually orthogonal idempotents is ad hoc.

Nevertheless, given a set of mutually orthogonal idempotents $\left\{c_{1}, \ldots, c_{n}\right\}$ on $H^{*}(\Sigma G / T ; \mathbb{Z} / p \mathbb{Z})$, by Lemma 4.2 we obtain a $p$-local homotopy equivalence

$$
\Sigma G / T \simeq \bigvee_{i=1}^{n} \operatorname{Tel}\left(c_{i}\right)
$$

4.3. Putting the two decompositions together. In general, if $p_{1}, \ldots, p_{n}$ and $q_{1}, \ldots, q_{m}$ are two sets of mutually orthogonal idempotents on $H^{*}(X ; \mathbb{Z} / p \mathbb{Z})$ that commute, where $X$ is a simplyconnected finite co-H-space, then the collection $\left\{p_{i} \circ q_{j} \mid 1 \leq i \leq n, 1 \leq j \leq m\right\}$ is another set of mutually orthogonal idempotents on $H^{*}(X ; \mathbb{Z} / p \mathbb{Z})$. In our case, the idempotents $\varphi_{i}^{*}$ from the unstable Adams operations and the idempotents $c_{j}^{*}$ from the action of the Weyl group commute since $\varphi_{i}^{*}$ is just a projection on to the subspaces consisting of elements of specific degrees while $c_{j}^{*}$ preserves the degrees. Thus the maps $\left\{\varphi_{i} \circ c_{j} \mid 1 \leq i \leq n, 1 \leq j \leq m\right\}$ form a set of mutually orthogonal idempotents on $H^{*}(\Sigma G / T ; \mathbb{Z} / p \mathbb{Z})$, and produce a finer decomposition of $\Sigma G / T$. We think of the decomposition based on the unstable Adams operation as splitting $H^{*}(\Sigma G / T ; \mathbb{Z} / p \mathbb{Z})$ "horizontally" while the one based on Weyl group action splits "vertically."

Note that as any space rationally splits into a wedge of spheres after suspension, we are primarily interested in $p$-local decompositions of $\Sigma G / T$ for a small prime $p$.

\section{Examples}

In identifying homotopy types of telescopes, we freely use the fact that the element $\eta \in \pi_{n+1}\left(S^{n}\right)$ is detected by the Steenrod operation $S q^{2}$ and at odd primes the element $\alpha_{1} \in \pi_{n+2 p-3}\left(S^{n}\right)$ is detected by the Steenrod operation $\mathcal{P}^{1}$. This is equivalent to saying that if $\tilde{H}^{*}\left(\operatorname{Tel}\left(c_{i}\right) ; \mathbb{Z} / 2 \mathbb{Z}\right) \cong \mathbb{Z} / 2 \mathbb{Z}\left\{x, S q^{2}(x)\right\}$ for $|x|=d \geq 3$ then there is a 2-local homotopy equivalence $\operatorname{Tel}\left(c_{i}\right) \simeq \Sigma^{d-2} \mathbb{C} P^{2}$, and if $p$ is odd and $\tilde{H}^{*}\left(\operatorname{Tel}\left(c_{i}\right) ; \mathbb{Z} / p \mathbb{Z}\right) \cong \mathbb{Z} / p \mathbb{Z}\left\{x, \mathcal{P}^{1}(x)\right\}$ for $|x|=d \geq 3$ then there is a $p$-local homotopy equivalence $\operatorname{Tel}\left(c_{i}\right) \simeq A(d, d+2 p-2)$ where $A(d, d+2 p-2)$ is the homotopy cofiber of $S^{d+2 p-3} \stackrel{\alpha_{1}}{\longrightarrow} S^{d}$.

In what follows, for a fixed prime $p$, we generically use the notation $c_{1}, \ldots, c_{n}$ for a set of mutually orthogonal idempotents in the group ring $\mathbb{Z} / p \mathbb{Z}[W]$ and let $V_{1}, \ldots, V_{n}$ be their images in $H^{*}(G / T ; \mathbb{Z} / p \mathbb{Z})$. By abuse of notation, we use the same symbol $c_{i}$ to denote the corresponding idempotents on $H^{*}(\Sigma G / T ; \mathbb{Z} / p \mathbb{Z})$. The action of the Steenrod operations $S q^{2}$ or $\mathcal{P}^{1}$ are determined by, for example, [7]. Computation on cohomology is carried out with the aid of a computer code described in [11]. Idempotents in the group ring are obtained by solving quadratic equations in prime fields.

5.1. Type $A_{n}$ case. For the type $A_{n}$-case, non-modular irreducible representations $V_{\lambda}$ are obtained by considering the Young symmetrizers for all the standard tableaux of shape $\lambda$. However, they are not always mutually orthogonal [21]. We will look at some low rank cases in an ad hoc way.

Example 5.1. Since $S U(2) / T=S^{2}$, the simplest non-trivial case is when $G=S U(3)$. By Theorems 2.2 and $2.3, H^{*}\left(S U(3) / T^{2} ; \mathbb{Z}\right) \simeq \mathbb{Z}\left[x_{1}, x_{2}, x_{3}\right] /\left(e_{1}, e_{2}, e_{3}\right) \simeq \mathbb{Z}\left\langle 1, \sigma_{1}, \sigma_{2}, \sigma_{12}, \sigma_{21}, \sigma_{121}\right\rangle$. It is well-known that the type- $A_{r}$ Weyl group is the symmetric group $S_{r+1}$. In particular, $W=\left\langle s_{1}, s_{2}\right\rangle$, where the simple reflection $s_{i}$ swaps $x_{i}$ and $x_{i+1}$. The Schubert cell decomposition 
looks like
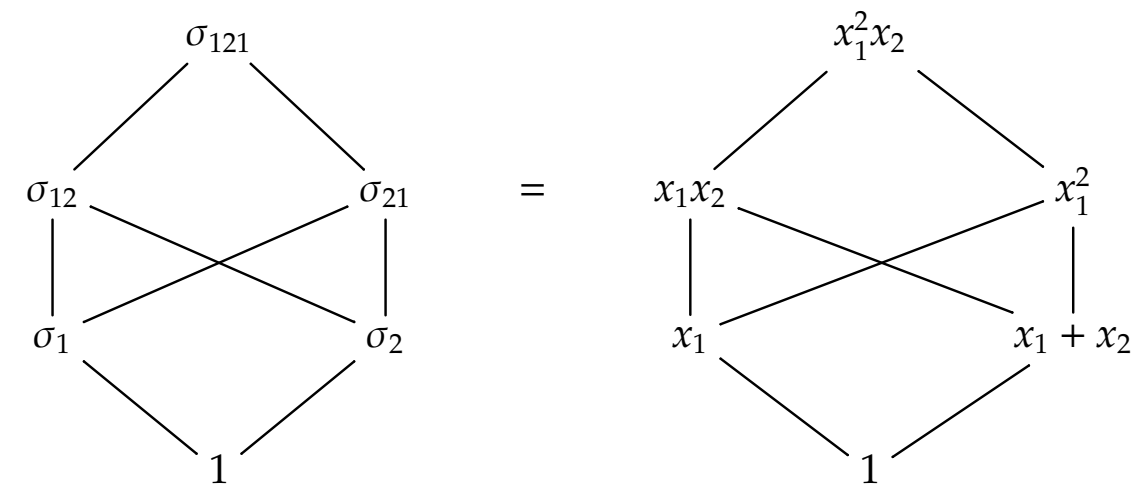

where $\sigma_{1}^{3}=\sigma_{2}^{3}=0$. The horizontal level indicates the degree of the cells, and the lines indicate possible non-trivial attaching maps.

We first find mutually orthogonal idempotents in the group ring (see $\S 4.2$ ). For $p=2$, there is a set of mutually orthogonal idempotents in $\mathbb{Z} / 2 \mathbb{Z}[W]$

$$
\begin{aligned}
& c_{1}=1+s_{12}+s_{21} \\
& c_{2}=1+s_{2}+s_{21}+s_{121} \\
& c_{3}=1+s_{2}+s_{12}+s_{121}
\end{aligned}
$$

satisfying

$$
\begin{aligned}
& V_{1}=\left\langle 1, \sigma_{121}\right\rangle \\
& V_{2}=\left\langle\sigma_{1}+\sigma_{2}, \sigma_{12}+\sigma_{21}\right\rangle \\
& V_{3}=\left\langle\sigma_{1}, \sigma_{21}\right\rangle
\end{aligned}
$$

where $\operatorname{Sq}^{2}\left(\sigma_{1}\right)=\sigma_{21}, \operatorname{Sq}^{2}\left(\sigma_{2}\right)=\sigma_{12}$. Therefore there is a 2-local homotopy equivalence

$$
\Sigma S U(3) / T^{2} \simeq_{2} S^{7} \vee \Sigma \mathbb{C} P^{2} \vee \Sigma \mathbb{C} P^{2} .
$$

For $p=3$, there is a set of mutually orthogonal idempotents in $\mathbb{Z} / 3 \mathbb{Z}[W]$

$$
\begin{aligned}
& c_{1}=2+s_{121} \\
& c_{2}=1-c_{1}
\end{aligned}
$$

satisfying

$$
\begin{aligned}
& V_{1}=\left\langle 1, \sigma_{1}+2 \sigma_{2}, \sigma_{12}+\sigma_{21}\right\rangle \\
& V_{2}=\left\langle\sigma_{1}+\sigma_{2}, \sigma_{12}+2 \sigma_{21}, \sigma_{121}\right\rangle
\end{aligned}
$$

where there is no non-trivial $\mathcal{P}^{1}$. Therefore each telescope is 3-locally homotopy equivalent to a wedge of spheres and we obtain a 3-local homotopy equivalence

$$
\Sigma S U(3) / T^{2} \simeq_{3} S^{3} \vee S^{3} \vee S^{5} \vee S^{5} \vee S^{7} .
$$

If $p>3$, for degree reasons the unstable Adams operations ( $\$ 4.1)$ imply that there is a $p$-local homotopy equivalence

$$
\Sigma S U(3) / T^{2} \simeq_{p} S^{3} \vee S^{3} \vee S^{5} \vee S^{5} \vee S^{7}
$$

The next example is $S U(4) / T^{3}$. It will be helpful to have some splitting information that comes from geometry as well as Adams operations and the action of the Weyl group.

Lemma 5.2. The stable normal bundle of any flag manifold $G / T$ is trivial. In particular, the top cell of $G / T$ stably splits off. 
Proof. Let $\mathfrak{g}$ and $\mathrm{t}$ be the Lie algebras of $G$ and $T$ respectively. Take a regular element $X \in \mathfrak{g}$ and consider the adjoint embedding $G / T \rightarrow \mathfrak{g}$ induced by $g \mapsto A d_{g}(X)$. The normal bundle $v$ is $G \times_{T} t$, which is trivial since the adjoint action of $T$ on $t$ is trivial. By the Pontrjagin-Thom construction combined with the pinching map, we obtain the splitting

$$
S^{\operatorname{dim}(G)} \rightarrow(G / T)^{v} \simeq \Sigma^{\operatorname{dim}(T)}(G / T)_{+} \rightarrow S^{\operatorname{dim}(G)},
$$

where $(G / T)^{v}$ is the Thom complex of the normal bundle $v$.

Example 5.3. The rank of $H^{*}\left(S U(4) / T^{3} ; \mathbb{Z}\right) \simeq \mathbb{Z}\left[x_{1}, x_{2}, x_{3}, x_{4}\right] /\left(e_{1}, e_{2}, e_{3}, e_{4}\right)$ is $\{1,3,5,6,5,3,1\}$. The Weyl group $W$ is the symmetric group $\left\langle s_{1}, s_{2}, s_{3}\right\rangle$ with $|W|=24$.

For $p=2$, there do not appear to be so many idempotents. For example, $c_{1}=s_{23}+s_{32}$ and $c_{2}=1-c_{1}$ form a mutually orthogonal set of idempotents in $\mathbb{Z} / 2 \mathbb{Z}[W]$ but they do not help much in terms of producing splittings with identifiable wedge summands.

For $p=3$, there is a set of mutually orthogonal idempotents in $\mathbb{Z} / 3 \mathbb{Z}[W]$

$$
\begin{aligned}
& c_{1}=\left(1+s_{1}+s_{2}+s_{12}+s_{21}+s_{121}\right)\left(1-s_{12321}\right) \\
& c_{2}=\left(1+s_{12321}\right)\left(1-s_{1}-s_{2}+s_{12}+s_{21}-s_{121}\right) \\
& c_{3}=2+2 s_{1}+2 s_{2}+2 s_{3}+2 s_{21}+2 s_{12}+2 s_{32}+2 s_{23}+2 s_{121}+s_{321}+2 s_{232}+s_{123}+s_{1321}+s_{2321}+s_{1213} \\
& +s_{1232}+s_{21321}+s_{12321}+s_{12132}+s_{121321} \\
& c_{4}=2+s_{2}+2 s_{13}+s_{213}+s_{132}+2 s_{2132}+s_{12321}+2 s_{121321} \\
& c_{5}=2+2 s_{2}+2 s_{13}+2 s_{213}+2 s_{132}+2 s_{2132}+212321+2 s_{121321} \\
& c_{6}=2+s_{1}+s_{2}+2 s_{3}+2 s_{21}+s_{13}+2 s_{12}+s_{32}+s_{23}+s_{121}+s_{321}+2 s_{213}+2 s_{132}+2 s_{232}+2 s_{123}+2 s_{1321} \\
& +2 s_{2321}+s_{1213}+s_{2132}+s_{1232}+s_{21321}+s_{12321}+2 s_{12132}+2 s_{121321} \\
& c_{7}=2+s_{23}+s_{32}+s_{3}+s_{13}+s_{213}+s_{132}+s_{232}+s_{2132}+2 s_{1213}+2 s_{12132}+2 s_{123}+2 s_{1232}+s_{1321}+s_{321} \\
& +s_{21321}+2 s_{121321}+s_{2321}+2 s_{12321}+2 s_{121}+2 s_{21}+2 s_{12}+2 s_{2}+2 s_{1} \\
& c_{8}=1-\sum_{i=1}^{7} c_{i}
\end{aligned}
$$

satisfying

$$
\begin{aligned}
& V_{1}=\left\langle\sigma_{3}, \sigma_{23}, \sigma_{123}\right\rangle \\
& V_{2}=\left\langle\sigma_{123}+\sigma_{121}+\sigma_{321}+2 \sigma_{213}+2 \sigma_{132}+\sigma_{232}, \sigma_{1213}+\sigma_{1232}+2 \sigma_{1321}+2 \sigma_{2321}, \sigma_{21321}+\sigma_{12321}+\sigma_{12132}\right\rangle \\
& V_{3}=\left\langle\sigma_{321}+2 \sigma_{213}+\sigma_{123}, \sigma_{2321}+2 \sigma_{1213}, \sigma_{12321}\right\rangle \\
& V_{4}=\left\langle 2 \sigma_{12}+\sigma_{13}+\sigma_{21}+2 \sigma_{32}+\sigma_{23}, 2 \sigma_{2321}+\sigma_{2132}+2 \sigma_{1213}, \sigma_{121321}\right\rangle \\
& V_{5}=\left\langle 1, \sigma_{12}+\sigma_{13}+\sigma_{23}, \sigma_{2321}+\sigma_{1213}\right\rangle \\
& V_{6}=\left\langle\sigma_{321}+2 \sigma_{213}+2 \sigma_{132}+2 \sigma_{232}+\sigma_{123}, \sigma_{2321}+\sigma_{1232}+2 \sigma_{2132}+2 \sigma_{1213}, \sigma_{12321}+2 \sigma_{12132}\right\rangle \\
& V_{7}=\left\langle\sigma_{1}+2 \sigma_{3}, \sigma_{21}+\sigma_{23}, \sigma_{321}+2 \sigma_{123}\right\rangle \\
& V_{8}=\left\langle\sigma_{1}+\sigma_{2}+\sigma_{3}, \sigma_{21}+2 \sigma_{12}+\sigma_{32}+2 \sigma_{23}, \sigma_{321}+2 \sigma_{132}+\sigma_{213}\right\rangle .
\end{aligned}
$$

The non-trivial actions of $\mathcal{P}^{1}$ are

$$
\begin{aligned}
V_{1}, V_{7}, V_{8}: H^{2} & \rightarrow H^{6} \\
V_{4}, V_{5}: H^{4} & \rightarrow H^{8} \\
V_{2}, V_{3}, V_{6}: H^{6} & \rightarrow H^{10} .
\end{aligned}
$$

For degree reasons in cohomology, the unstable Adams operations split $\operatorname{Tel}\left(c_{i}\right)$ for $i \in\{1,7,8\}$ into wedge summands: one inheriting the degree 3 and 7 generators in cohomology and the other inheriting the degree 5 generator. Similarly, the unstable Adams operations split $\operatorname{Tel}\left(c_{i}\right)$ for $i \in\{2,3,6\}$ into wedge summands: one inheriting the degree 7 and 11 generators in 
cohomology and the other inheriting the degree 9 generator. All together we obtain 3-local homotopy equivalences

$$
\begin{aligned}
& \operatorname{Tel}\left(c_{1}\right) \simeq \operatorname{Tel}\left(c_{7}\right) \simeq \operatorname{Tel}\left(c_{8}\right) \simeq A(3,7) \vee S^{5} \\
& \operatorname{Tel}\left(c_{2}\right) \simeq \operatorname{Tel}\left(c_{3}\right) \simeq \operatorname{Tel}\left(c_{6}\right) \simeq A(7,11) \vee S^{9}
\end{aligned}
$$

Note that $\mathrm{Tel}\left(c_{5}\right) \simeq A(5,9)$ and $\mathrm{Tel}\left(c_{4}\right)$ is a three-cell complex whose 9-skeleton is $A(5,9)$. We claim that $\operatorname{Tel}\left(c_{4}\right) \simeq A(5,9) \vee S^{13}$. To see this we show that the attaching map $g: S^{12} \longrightarrow A(5,9)$ for the top cell is null homotopic. Since there is no Steenrod operation connecting the 9 and 13-cells in $\mathrm{Tel}\left(c_{4}\right)$, the composition $S^{12} \stackrel{g}{\longrightarrow} A(5,9) \stackrel{q}{\longrightarrow} S^{9}$ must be null homotopic, where $q$ is the pinch map to the top cell. Therefore $g$ lifts to the homotopy fibre of $q$, which by a Serre spectral sequence argument, is homotopy equivalent to $S^{5}$ in dimensions $\leq 12$. Thus $g$ is homotopic to a composite $S^{12} \stackrel{t \cdot \alpha_{2}}{\longrightarrow} S^{5} \hookrightarrow A(5.9)$, where $\alpha_{2}$ generates $\pi_{12}\left(S^{5}\right) \cong \mathbb{Z} / 3 \mathbb{Z}$ and $t \in \mathbb{Z} / 3 \mathbb{Z}$. Each map in this composite is stable, so $g$ is stable. But by Lemma 5.2, the top cell of Tel $\left(c_{4}\right)$ splits off stably. Thus $g$ is stably trivial, and so $g$ itself must be trivial. Hence, we have $\operatorname{Tel}\left(c_{4}\right) \simeq A(5,9) \vee S^{13}$. To sum up, there is a 3-local homotopy equivalence

$$
\Sigma S U(4) / T^{3} \simeq_{3} 3 A(3,7) \vee 3 S^{5} \vee 3 A(7,11) \vee 3 S^{9} \vee 2 A(5,9) \vee S^{13}
$$

If $p=5$ then for degree reasons the unstable Adams operations decompose $\Sigma S U(4) / T^{3}$ as a wedge $X_{1} \vee \cdots \vee X_{4}$ where $H^{*}(X ; \mathbb{Z} / 5 \mathbb{Z})$ consists of the degree 3 and 11 elements in $H^{*}\left(S U(4) / T^{3} ; \mathbb{Z} / 5 \mathbb{Z}\right), H^{*}\left(X_{2} ; \mathbb{Z} / 5 \mathbb{Z}\right)$ consists of the degree 5 and 13 elements, $H^{*}\left(X_{3} ; \mathbb{Z} / 5 \mathbb{Z}\right)$ consists of the degree 7 elements, and $H^{*}\left(X_{4} ; \mathbb{Z} / 5 \mathbb{Z}\right)$ consists of the degree 9 elements. On the other hand, since $\sigma_{w}^{5}=0$ for all $l(w)=1$, all $\mathcal{P}^{1}$ are trivial. Thus there is a 5-local homotopy equivalence

$$
\Sigma S U(4) / T^{3} \simeq_{5} 3 S^{3} \vee 5 S^{5} \vee 6 S^{7} \vee 5 S^{9} \vee 3 S^{11} \vee S^{13}
$$

If $p>5$ then for degree reasons the unstable Adams operations decompose $S U(4) / T^{3}$ as a wedge of spheres (the same wedge as in the $p=5$ case.)

5.2. Type $B_{2}=C_{2}$. The Weyl group $W$ is the hyper-octahedral group $\left\langle s_{1}, s_{2}\right\rangle$ with $|W|=8$. We have $H^{*}(S p(2) / T)=\frac{\mathbb{Z}\left[x_{1}, x_{2}\right]}{\left(x_{1}^{2}+x_{2}^{2}, x_{1}^{2} x_{2}^{2}\right)}$ and its Betti numbers are $\{1,2,2,2,1\}$.

For $p=2$, there is no non-trivial idempotents.

For $p>2$ (the non-modular case), there is a set of mutually orthogonal idempotents in $\mathbb{Z} / p \mathbb{Z}[W]$

$$
\begin{aligned}
& c_{1}=\frac{1}{8}\left(1+s_{1}-s_{2}-s_{12}-s_{21}-s_{121}+s_{212}+s_{1212}\right) \\
& c_{2}=\frac{1}{8}\left(1-s_{1}+s_{2}-s_{12}-s_{21}+s_{121}-s_{212}+s_{1212}\right) \\
& c_{3}=\frac{1}{8}\left(1-s_{1}-s_{2}+s_{12}+s_{21}-s_{121}-s_{212}+s_{1212}\right) \\
& c_{4}=\frac{1}{8}\left(1-s_{1}+s_{212}-s_{1212}\right) \\
& c_{5}=\frac{1}{8}\left(1+s_{1}-s_{212}-s_{1212}\right) \\
& c_{6}=\frac{1}{8} \sum_{w \in W} w
\end{aligned}
$$


satisfying

$$
\begin{aligned}
& V_{1}=\left\langle\sigma_{12}\right\rangle \\
& V_{2}=\left\langle\sigma_{21}\right\rangle \\
& V_{3}=\left\langle\sigma_{1212}\right\rangle \\
& V_{4}=\left\langle\sigma_{1}-\sigma_{2}, \sigma_{121}+\sigma_{212}\right\rangle \\
& V_{5}=\left\langle\sigma_{2}, \sigma_{212}\right\rangle \\
& V_{6}=\langle 1\rangle
\end{aligned}
$$

where $\sigma_{1}^{3}=\sigma_{121}$ and $\sigma_{2}^{3}=2 \sigma_{212}$. In particular, we obtain $\operatorname{Tel}\left(c_{1}\right) \simeq \operatorname{Tel}\left(c_{2}\right) \simeq S^{5}, \operatorname{Tel}\left(c_{3}\right) \simeq S^{9}$ and $\operatorname{Tel}\left(c_{6}\right) \simeq *$.

If $p=3$ then the equations $\sigma_{1}^{3}=\sigma_{121}$ and $\sigma_{2}^{3}=2 \sigma_{212}$ imply that $\mathcal{P}^{1}$ is non-trivial on $\sigma_{1}$ and $\sigma_{2}$. Therefore $\operatorname{Tel}\left(c_{4}\right) \simeq \operatorname{Tel}\left(c_{5}\right) \simeq A(3,7)$. Hence there is a 3-local homotopy equivalence

$$
\Sigma S p(2) / T \simeq_{3} 2 A(3,7) \vee 2 S^{5} \vee S^{9} .
$$

If $p \geq 5$ then all Steenrod operations $\mathcal{P}^{1}$ are trivial so $\operatorname{Tel}\left(c_{4}\right) \simeq \operatorname{Tel}\left(c_{5}\right) \simeq S^{3} \vee S^{7}$. Hence there is a $p$-local homotopy equivalence

$$
\Sigma S p(2) / T \simeq_{p} 2 S^{3} \vee 2 S^{5} \vee 2 S^{7} \vee S^{9}
$$

5.3. Type $G_{2}$. The Weyl group $W$ is the dihedral group $D_{6}=\left\langle s_{1}, s_{2}\right\rangle$ with $|W|=12$. The cohomology of $G_{2} / T$ is computed by $[5,23]$ as

$$
H^{*}\left(G_{2} / T\right)=\frac{\mathbb{Z}\left[x_{1}, x_{2}, x_{3}, \gamma\right]}{\left(e_{1}, e_{2}, e_{3}-2 \gamma, \gamma^{2}\right)}
$$

For $p=2$, there is a set of mutually orthogonal idempotents in $\mathbb{Z} / 2 \mathbb{Z}[W]$

$$
\begin{aligned}
& c_{1}=1+s_{1212}+s_{2121} \\
& c_{2}=1+s_{212}+s_{1212}+s_{12121} \\
& c_{3}=1+s_{212}+s_{2121}+s_{12121}
\end{aligned}
$$

satisfying

$$
\begin{aligned}
& V_{1}=\left\langle 1, \sigma_{121}, \sigma_{212}, \sigma_{121212}\right\rangle, \\
& V_{2}=\left\langle\sigma_{1}+\sigma_{2}, \sigma_{12}+\sigma_{21}, \sigma_{1212}+\sigma_{2121}, \sigma_{12121}+\sigma_{21212}\right\rangle, \\
& V_{3}=\left\langle\sigma_{1}, \sigma_{21}, \sigma_{2121}, \sigma_{12121}\right\rangle,
\end{aligned}
$$

where $\operatorname{Sq}^{2}\left(\sigma_{1}\right)=\sigma_{21}, \operatorname{Sq}^{2}\left(\sigma_{2}\right)=\sigma_{12}, \operatorname{Sq}^{2}\left(\sigma_{1212}\right)=\sigma_{21212}, \operatorname{Sq}^{2}\left(\sigma_{2121}\right)=\sigma_{12121}$. The multiple generators of different degrees in the modules $V_{i}$ imply that the telescopes of the maps $c_{i}$ are not readily identifiable. So we say nothing more than there is a 2-local homotopy equivalence

$$
\Sigma G_{2} / T \simeq_{2} \operatorname{Tel}\left(c_{1}\right) \vee \operatorname{Tel}\left(c_{2}\right) \vee \operatorname{Tel}\left(c_{3}\right) .
$$

For $p=3$, there is a set of mutually orthogonal idempotents in $\mathbb{Z} / 3 \mathbb{Z}[W]$

$$
\begin{aligned}
& c_{1}=1+s_{1}+s_{21212}+s_{121212} \\
& c_{2}=1+s_{1}-s_{21212}-s_{121212} \\
& c_{3}=1-s_{1}-s_{21212}+s_{121212} \\
& c_{4}=1-s_{1}+s_{21212}-s_{121212}
\end{aligned}
$$

satisfying

$$
\begin{aligned}
& V_{1}=\left\langle 1, \sigma_{12}, \sigma_{1212}\right\rangle \\
& V_{2}=\left\langle\sigma_{2}, \sigma_{212}, \sigma_{21212}\right\rangle \\
& V_{3}=\left\langle\sigma_{21}+\sigma_{12}, \sigma_{2121}-\sigma_{1212}, \sigma_{121212}\right\rangle \\
& V_{4}=\left\langle\sigma_{1}+\sigma_{2}, \sigma_{121}, \sigma_{12121}-\sigma_{21212}\right\rangle
\end{aligned}
$$


The non-trivial actions of $\mathcal{P}^{1}$ are

$$
\begin{aligned}
V_{1}, V_{3}: H^{4} & \rightarrow H^{8} \\
V_{2}: H^{6} & \rightarrow H^{10} \\
V_{4}: H^{2} & \rightarrow H^{6} .
\end{aligned}
$$

In particular, $\operatorname{Tel}\left(c_{1}\right) \simeq A(5,9)$, but the other telescopes are not as readily identifiable. So we say nothing more right now other than there is a 3-local homotopy equivalence

$$
\Sigma G_{2} / T \simeq_{3} A(5,9) \vee \operatorname{Tel}\left(c_{2}\right) \vee \operatorname{Tel}\left(c_{3}\right) \vee \operatorname{Tel}\left(c_{4}\right) .
$$

For $p>3$ (the non-modular case), the regular representation decomposes into four 1dimensional and four 2-dimensional irreducible representations. The maps inducing the cohomology idempotents corresponding to the 1-dimensional irreducible representations are given by

$$
\begin{array}{ll}
c_{1}=\frac{1}{12} \sum_{w \in W} w & c_{2}=\frac{1}{12} \sum_{w \in W}(-1)^{l(w)} w \\
c_{3}=\frac{1}{12} \sum_{w \in W}(-1)^{\#_{w} 1} w & c_{4}=\frac{1}{12} \sum_{w \in W}(-1)^{\#_{w} 2} w
\end{array}
$$

where $\#_{w} i$ is the number of $s_{i}{ }^{\prime}$ s in $w$, and these satisfy

$$
\begin{array}{lc}
V_{1}=\langle 1\rangle & V_{2}=\left\langle\sigma_{121212}\right\rangle \\
V_{3}=\left\langle\sigma_{121}\right\rangle & V_{4}=\left\langle\sigma_{212}\right\rangle .
\end{array}
$$

In particular, we obtain $\operatorname{Tel}\left(c_{1}\right) \simeq * \operatorname{Tel}\left(c_{2}\right) \simeq S^{13}$ and $\operatorname{Tel}\left(c_{3}\right) \simeq \operatorname{Tel}\left(c_{4}\right) \simeq S^{7}$.

The maps inducing the cohomology idempotents corresponding to the 2-dimensional irreducible representations are given by

$$
\begin{aligned}
& c_{5}=\frac{1}{12}\left(2+s_{1}-2 s_{2}-s_{12}-s_{21}+s_{121}+s_{212}-s_{1212}-s_{2121}-2 s_{12121}+s_{21212}+2 s_{121212}\right) \\
& c_{6}=\frac{1}{12}\left(2-s_{1}+2 s_{2}-s_{12}-s_{21}-s_{121}-s_{212}-s_{1212}-s_{2121}+2 s_{12121}-s_{21212}+2 s_{121212}\right) \\
& c_{7}=\frac{1}{6}\left(1-s_{1}-s_{2}+s_{21}-s_{1212}+s_{12121}+s_{21212}-s_{121212}\right) \\
& c_{8}=\frac{1}{6}\left(1+s_{1}+s_{2}+s_{12}-s_{2121}-s_{12121}-s_{21212}-s_{121212}\right)
\end{aligned}
$$

and these satisfy

$$
\begin{aligned}
& V_{5}=\left\langle 2 \sigma_{12}-\sigma_{21}, 2 \sigma_{1212}+\sigma_{2121}\right\rangle \\
& V_{6}=\left\langle\sigma_{21}, \sigma_{2121}\right\rangle \\
& V_{7}=\left\langle 3 \sigma_{1}-2 \sigma_{2}, 3 \sigma_{12121}+2 \sigma_{21212}\right\rangle \\
& V_{8}=\left\langle\sigma_{2}, \sigma_{21212}\right\rangle .
\end{aligned}
$$

If $p=5$ then the Steenrod operation $\mathcal{P}^{1}$ is trivial on $V_{5}$ and $V_{6}$ for degree reasons. So Tel $\left(c_{5}\right) \simeq$ $\operatorname{Tel}\left(c_{6}\right) \simeq S^{5} \vee S^{9}$. On the other hand, $\mathcal{P}^{1}\left(\sigma_{2}\right)=3 \sigma_{21212}$ and $\mathcal{P}^{1}\left(3 \sigma_{1}-2 \sigma_{2}\right)=2\left(3 \sigma_{12121}+2 \sigma_{21212}\right)$ so $\operatorname{Tel}\left(c_{7}\right) \simeq \operatorname{Tel}\left(c_{8}\right) \simeq A(3,11)$. Therefore there is a 5-local homotopy equivalence

$$
\Sigma G_{2} / T \simeq_{5} 2 S^{5} \vee 2 S^{7} \vee 2 S^{9} \vee S^{13} \vee 2 A(3,11) .
$$

If $p>5$ then the Steenrod operation $\mathcal{P}^{1}$ is trivial on each of $V_{5}, V_{6}, V_{7}$ and $V_{8}$. Therefore there is a $p$-local homotopy equivalence

$$
\Sigma G_{2} / T \simeq_{p} 2 S^{3} \vee 2 S^{5} \vee 2 S^{7} \vee 2 S^{9} \vee 2 S^{11} \vee S^{13}
$$


Remark 5.4. It is interesting to note that factors are "Poincaré dual" to each other. For example, $\left(V_{6}, V_{7}\right)$ in $S U(4) / T$ with $p=3$ are dual to each other in the sense that the generators in the complimentary degrees multiply to the top degree element (e.g., $\left(\sigma_{2321}+\sigma_{1232}+2 \sigma_{2132}+\right.$ $\left.\left.2 \sigma_{1213}\right)\left(\sigma_{21}+\sigma_{23}\right)=\sigma_{121321} \bmod 3\right)$. For $S U(4) / T$ with $p=3,\left(V_{1}, V_{2}\right),\left(V_{3}, V_{8}\right)$, and $\left(V_{4}, V_{5}\right)$ are all dual pairs as well. For $\operatorname{Sp}(2) / T$ with $p>2,\left(V_{1}, V_{2}\right),\left(V_{3}, V_{6}\right)$, and $\left(V_{4}, V_{5}\right)$ are dual pairs. For $G_{2} / T$ with $p>3,\left(V_{1}, V_{2}\right),\left(V_{3}, V_{4}\right),\left(V_{5}, V_{6}\right)$, and $\left(V_{7}, V_{8}\right)$ are dual pairs. It might be interesting to find a geometric explanation for this.

\section{SElF-MAPS OF FLAG MANifOldS}

The decomposition of $\Sigma G / T$ allows for a calculation of the factor [G/T,G] of [G/T,G/T]. Since $G$ is a topological group, it has a classifying space $B G$, and $G \simeq \Omega B G$. Therefore there is an adjunction giving an isomorphism of groups $[G / T, G] \cong[G / T, \Omega B G] \cong[\Sigma G / T, B G]$. Suppose that there is a homotopy decomposition $\Sigma G / T \simeq \bigvee_{i=1}^{k} A_{i}$. As this is a decomposition of spaces rather than co- $H$-spaces, there is a set isomorphism $[\Sigma G / T, B G] \cong\left[\bigvee_{i=1}^{k} A_{i}, B G\right] \cong$ $\prod_{i=1}^{k}\left[A_{i}, B G\right]$. Combining these isomorphisms gives the following.

Lemma 6.1. If $\Sigma G / T \simeq \bigvee_{i=1}^{k} A_{i}$ then there is an isomorphism of sets $[G / T, G] \cong \prod_{i=1}^{k}\left[A_{i}, B G\right]$.

Another useful general lemma is the following.

Lemma 6.2. If $G$ is simply-connected then, rationally, $[G / T, G] \cong 0$. Consequently, $[G / T, G]$ is the product of its $p$-components for all primes $p$.

Proof. In all cases, there is a rational homotopy equivalence $G \simeq \prod_{i=1}^{m} K\left(\mathbb{Q}, 2 n_{i}-1\right)$ for some sequence $\left\{i_{1}, \ldots, i_{m}\right\}$. Thus $[G / T, G] \cong \prod_{i=1}^{m} H^{2 n_{i}-1}(G / T ; \mathbb{Q})$. But $H^{\text {odd }}(G / T ; \mathbb{Q}) \cong 0$, so we obtain a rational isomorphism $[G / T, G] \cong 0$.

We now explicitly calculate $[G / T, G]$ when $G$ is one of $S U(3), S U(4), S p(2)$ or $G_{2}$.

Proposition 6.3. There is a group isomorphism $\left[S U(3) / T^{2}, S U(3)\right] \cong \mathbb{Z} / 6 \mathbb{Z}$ and the generator corresponds to the self-map

$$
S U(3) / T^{2} \stackrel{q}{\longrightarrow} S^{6} \stackrel{f}{\longrightarrow} S U(3) \longrightarrow S U(3) / T
$$

where $q$ is the pinch to the top cell and $f$ represents the generator of $\pi_{6}(S U(3)) \cong \mathbb{Z} / 6 \mathbb{Z}$.

Proof. By Lemma 6.2, to calculate $\left[S U(3) / T^{2}, S U(3)\right]$ it suffices to localize and work prime by prime.

Case 1: $p=2$. By Example 5.1, there is a 2-local homotopy equivalence

$$
\Sigma S U(3) / T^{2} \simeq S^{7} \vee \Sigma \mathbb{C} P^{2} \vee \Sigma \mathbb{C} P^{2} \text {. }
$$

Therefore

$$
\begin{aligned}
{\left[\operatorname{SU}(3) / T^{2}, \operatorname{SU}(3)\right] } & \cong\left[\Sigma S U(3) / T^{2}, B S U(3)\right] \\
& \cong\left[S^{7}, B S U(3)\right] \times\left[\Sigma \mathbb{C} P^{2}, B S U(3)\right] \times\left[\Sigma \mathbb{C} P^{2}, B S U(3)\right] .
\end{aligned}
$$

By [16], the 2-component of $\pi_{6}(S U(3))$ is $\mathbb{Z} / 2 \mathbb{Z}$. Since the homotopy fibre of BSU(3) $\longrightarrow$ $\operatorname{BSU}(\infty)$ is 6-connected and $\Sigma \mathbb{C} P^{2}$ is 5 -dimensional we have

$$
\left[\Sigma \mathbb{C} P^{2}, B S U(3)\right] \cong\left[\Sigma \mathbb{C} P^{2}, B S U(\infty)\right] \cong \widetilde{K}\left(\Sigma \mathbb{C} P^{2}\right) \cong 0
$$

where $\widetilde{K}$ is reduced complex $K$-theory. Therefore

$$
\left[S U(3) / T^{2}, S U(3)\right] \cong \mathbb{Z} / 2 \mathbb{Z}
$$

and this corresponds to a nontrivial 2-local self-map of $S U(3) / T^{2}$ given by the composite

$$
S U(3) / T^{2} \stackrel{q}{\longrightarrow} S^{6} \stackrel{f}{\longrightarrow} S U(3) \longrightarrow S U(3) / T^{2}
$$

where $q$ is the pinch to the top cell and $f$ represents the generator of $\pi_{6}(S U(3)) \cong \mathbb{Z} / 2 \mathbb{Z}$. 
Case 2: $p>2$. By Example 5.1, localized at a prime $p>2$ there is a homotopy equivalence $\Sigma S U(3) / T^{2} \simeq 2 S^{3} \vee 2 S^{5} \vee S^{7}$. Therefore, by Lemma 6.1,

$$
\begin{aligned}
{\left[S U(3) / T^{2}, S U(3)\right] } & \cong 2\left[S^{3}, B S U(3)\right] \times 2\left[S^{5}, B S U(3)\right] \times\left[S^{7}, B S U(3)\right] \\
& \cong 2 \pi_{2}(\operatorname{SU}(3)) \times 2 \pi_{4}(\operatorname{SU}(3)) \times \pi_{6}(\operatorname{SU}(3)) .
\end{aligned}
$$

By [17], $\pi_{2}(S U(3)) \cong 0, \pi_{4}(S U(3)) \cong 0$ and after inverting $2, \pi_{6}(S U(3)) \cong \mathbb{Z} / 3 \mathbb{Z}$. Therefore, localized at $p>3$ we have $\left[S U(3) / T^{2}, S U(3)\right] \cong 0$ and localized at 3 we have

$$
\left[S U(3) / T^{2}, S U(3)\right] \cong \mathbb{Z} / 3 \mathbb{Z} \text {. }
$$

In the latter case, the generator corresponds to a nontrivial 3-local self-map of $S U(3) / T^{2}$ given by the composite

$$
S U(3) / T^{2} \stackrel{q}{\longrightarrow} S^{6} \stackrel{f}{\longrightarrow} S U(3) \longrightarrow S U(3) / T^{2}
$$

where $q$ is the pinch map to the top cell and $f$ represents a generator of $\pi_{6}(S U(3)) \cong \mathbb{Z} / 3 \mathbb{Z}$.

Combining both cases, we obtain a set isomorphism $\left[S U(3) / T^{2}, S U(3)\right] \cong \mathbb{Z} / 6 \mathbb{Z}$. To upgrade this to an isomorphism of groups, it suffices to show that the group $\left[S U(3) / T^{2}, \operatorname{SU}(3)\right]$ has an element of order 6 . But observe that the generators of the 2 and 3 -components are obtained from the same map

$$
S U(3) / T^{2} \stackrel{q}{\longrightarrow} S^{6} \stackrel{f}{\longrightarrow} S U(3) \longrightarrow S U(3) / T^{2}
$$

where $f$ represents a generator of $\pi_{6}\left(S^{3}\right) \cong \mathbb{Z} / 6 \mathbb{Z}$. Thus $f \circ q$ has order 6 in $\left[S U(3) / T^{2}, \operatorname{SU}(3)\right]$ and we are done.

Proposition 6.4. Localized away from 2 there is a set isomorphism $\left[S U(4) / T^{3}, S U(4)\right] \cong$ $\mathbb{Z} / 15 \mathbb{Z} \times 3(\mathbb{Z} / 5 \mathbb{Z})$. The group $\left[S U(4) / T^{3}, S U(4)\right]$ has a subgroup of order 15 corresponding to the self-map

$$
S U(4) / T^{3} \stackrel{q}{\longrightarrow} S^{12} \stackrel{f}{\longrightarrow} S U(4) \longrightarrow S U(4) / T^{3}
$$

where $q$ is the pinch map to the top cell and $f$ represents the 3 and 5-components of $\pi_{12}(S U(4)) \cong \mathbb{Z} / 60 \mathbb{Z}$. The group $\left[S U(4) / T^{3}, S U(4)\right]$ has three subgroups of order 5 corresponding to 5-local self-maps

$$
S U(4) / T^{3} \stackrel{q^{\prime}}{\longrightarrow} 3 S^{10} \vee S^{12} \stackrel{p_{i}}{\longrightarrow} S^{10} \stackrel{f_{i}}{\longrightarrow} S U(4) \longrightarrow S U(4) / T^{3}
$$

where $q^{\prime}$ collapses the 9-skeleton of $S U(4) / T^{3}$ to a point, and for $1 \leq i \leq 3$ the map $p_{i}$ pinches to the $i^{\text {th }}$-copy of $S^{10}$ while $f_{i}$ represents the generator of $\pi_{10}(S U(4)) \cong \mathbb{Z} / 5 \mathbb{Z}$.

Proof. By Lemma 6.2, to calculate $\left[S U(4) / T^{3}, S U(4)\right]$ it suffices to localize and work prime by prime.

Case 1: $p=3$. By Example 5.3, there is a 3-local homotopy equivalence

$$
\Sigma S U(4) / T^{3} \simeq 3 A(3,7) \vee 3 S^{5} \vee 3 A(7,11) \vee 3 S^{9} \vee 2 A(5,9) \vee S^{13} .
$$

We calculate $\left[\Sigma S U(4) / T^{3}, B S U(4)\right]$ by using Lemma 6.1 .

By [17], $\pi_{m}(B S U(4)) \cong 0$ for $m \in\{3,5,7,9\}$, so $[A(3,7), B S U(4)] \cong\left[S^{5}, B S U(4)\right] \cong\left[S^{9}, B S U(4)\right] \cong$ 0 , and $\pi_{13}(B S U(4)) \cong \mathbb{Z} / 60 \mathbb{Z}$. It remains to consider $[A(7,11), B S U(4)]$ and $[A(5,9), B S U(4)]$.

For $A(7,11)$, the cofibration sequence $S^{7} \longrightarrow A(7,11) \longrightarrow S^{11} \stackrel{\alpha_{1}}{\longrightarrow} S^{8}$ induces an exact sequence

$$
\left[S^{8}, B S U(4)\right] \stackrel{\left(\alpha_{1}\right)^{*}}{\longrightarrow}\left[S^{11}, B S U(4)\right] \longrightarrow[A(7,11), B S U(4)] \longrightarrow\left[S^{7}, B S U(4)\right] .
$$

On the one hand, $\left[S^{7}, B S U(4)\right]=\pi_{6}(S U(4)) \cong 0$. On the other hand, by [17], $\left[S^{8}, B S U(4)\right] \cong \mathbb{Z}$, $\left[S^{11}, B S U(4)\right] \cong \mathbb{Z} / 3 \mathbb{Z}$, and $\left(\alpha_{1}\right)^{*}$ is an epimorphism. Thus $[A(7,11), B S U(4)] \cong 0$.

For $A(5,9)$, the cofibration sequence $S^{5} \longrightarrow A(5,9) \longrightarrow S^{9} \stackrel{\alpha_{1}}{\longrightarrow} S^{6}$ induces an exact sequence

$$
\left[S^{6}, \operatorname{BSU}(4)\right] \stackrel{\left(\alpha_{1}\right)^{*}}{\longrightarrow}\left[S^{9}, \operatorname{BSU}(4)\right] \longrightarrow[A(5,9), \operatorname{BSU}(4)] \longrightarrow\left[S^{5}, \operatorname{BSU}(4)\right]
$$


On the one hand, $\left[S^{5}, B S U(4)\right]=\pi_{4}(S U(4)) \cong 0$. On the other hand, by [17], $\left[S^{6}, B S U(4)\right] \cong \mathbb{Z}$, $\left[S^{9}, B S U(4)\right] \cong \mathbb{Z} / 3 \mathbb{Z}$, and $\left(\alpha_{1}\right)^{*}$ is an epimorphism. Thus $[A(5,9), B S U(4)] \cong 0$.

Therefore, from (4) we obtain a set isomorphism

$$
\left[S U(4) / T^{3}, S U(4)\right] \cong \mathbb{Z} / 3 \mathbb{Z} \text {. }
$$

This is in fact a group isomorphism. It suffices to find an element of $\left[S U(4) / T^{3}, S U(4)\right]$ whose order when localized at 3 is 3 . But this is given by the map $q \circ f$ in the composite

$$
S U(4) / T^{3} \stackrel{q}{\longrightarrow} S^{12} \stackrel{f}{\longrightarrow} S U(4) \longrightarrow S U(4) / T^{3}
$$

where $q$ is the pinch map to the top cell and $f$ represents the 3-component of $\pi_{12}(S U(4)) \cong$ $\mathbb{Z} / 60 \mathbb{Z}$. The map $f \circ q$ has order 3 in $\left[S U(4) / T^{3}, S U(4)\right]$ so

Case 2: $p \geq 5$. By Example 5.3, localized at a prime $p \geq 5$ there is a homotopy equivalence

$$
\Sigma S U(4) / T^{3} \simeq 3 S^{3} \vee 5 S^{5} \vee 6 S^{7} \vee 5 S^{9} \vee 3 S^{11} \vee S^{13} \text {. }
$$

Therefore, by Lemma 6.1,

$$
\begin{array}{r}
{\left[S U(4) / T^{3}, \operatorname{SU}(4)\right] \cong 3\left[S^{3}, B S U(4)\right] \times 5\left[S^{5}, B S U(4)\right] \times 6\left[S^{7}, B S U(4)\right] \times} \\
5\left[S^{9}, B S U(4)\right] \times 3\left[S^{11}, B S U(4)\right] \times\left[S^{13}, B S U(4)\right] .
\end{array}
$$

By [17], $\pi_{2}(S U(4)) \cong 0, \pi_{4}(S U(4)) \cong 0, \pi_{6}(S U(4)) \cong 0$, and after inverting 2 and $3, \pi_{8}(S U(4)) \cong$ $0, \pi_{10}(S U(4)) \cong \mathbb{Z} / 5 \mathbb{Z}$ and $\pi_{12}(S U(4)) \cong \mathbb{Z} / 5 \mathbb{Z}$. Thus

$$
\left[S U(4) / T^{3}, S U(4)\right] \cong 4(\mathbb{Z} / 5 \mathbb{Z})
$$

and the generators correspond to two types of nontrivial 5-local self-maps. First,

$$
S U(4) / T^{3} \stackrel{q^{\prime}}{\longrightarrow} 3 S^{10} \vee S^{12} \stackrel{p_{i}}{\longrightarrow} S^{10} \stackrel{f_{i}}{\longrightarrow} S U(4) \longrightarrow S U(4) / T^{3}
$$

where $q^{\prime}$ is the map that collapses out the 9-skeleton of $S U(4) / T^{3}$, and for $1 \leq i \leq 3$ the map $p_{i}$ pinches to the $i^{t h}$-copy of $S^{10}$ while $f_{i}$ represents the generator of $\pi_{10}(S U(4)) \cong \mathbb{Z} / 5 \mathbb{Z}$. Second,

$$
S U(4) / T^{3} \stackrel{q}{\longrightarrow} S^{12} \stackrel{f}{\longrightarrow} S U(4) \longrightarrow S U(4) / T^{3}
$$

where $q$ is the pinch map to the top cell and $f$ represents the generator of $\pi_{12}(S U(4)) \cong \mathbb{Z} / 5 \mathbb{Z}$.

Finally, notice that the same map $S U(4) / T^{3} \stackrel{q}{\longrightarrow} S^{12} \stackrel{f}{\longrightarrow} S U(4)$ appears in the $p=3$ and $p=5$ cases, so $f \circ q$ has order 15 and generates a subgroup of order 15 in $\left[S U(4) / T^{3}, S U(4)\right]$.

Proposition 6.5. There is a group isomorphism $\left[G_{2} / T, G_{2}\right] \cong 0$.

Proof. By Lemma 6.2, to calculate $\left[G_{2} / T, G_{2}\right]$ it suffices to localize and work prime by prime.

Case 1: $p=2$. As in Section 5.3, there is a 2-local homotopy equivalence

$$
\Sigma G_{2} / T \simeq \operatorname{Tel}\left(c_{1}\right) \vee \operatorname{Tel}\left(c_{2}\right) \vee \operatorname{Tel}\left(c_{3}\right) .
$$

By Lemma 6.1, to calculate the 2-component of $\left[G_{2} / T, G_{2}\right]$ it is equivalent to calculate [Tel $\left.\left(c_{i}\right), B G_{2}\right]$ for $1 \leq i \leq 3$. The space Tel $\left(c_{1}\right)$ has cells in dimensions 7 and 13. By [16], $\pi_{7}\left(B G_{2}\right) \cong \pi_{13}\left(B G_{2}\right) \cong 0$, so [ $\left.\operatorname{Tel}\left(c_{1}\right), B G_{2}\right] \cong 0$. The spaces $\operatorname{Tel}\left(c_{2}\right)$ and $\operatorname{Tel}\left(c_{3}\right)$ both have cells in dimensions $3,5,9,11$, and the Steenrod operation $S q^{2}$ connects the 3 and 5 cells, and the 9 and 11 cells. Therefore, for $2 \leq i \leq 3$ there is a homotopy cofibration $\Sigma \mathbb{C} P^{2} \longrightarrow \operatorname{Tel}\left(c_{i}\right) \longrightarrow \Sigma^{7} \mathbb{C} P^{2}$. Let $g: \operatorname{Tel}\left(c_{i}\right) \longrightarrow B G_{2}$ be any map. By [16], $\pi_{3}\left(B G_{2}\right) \cong \pi_{5}\left(B G_{2}\right) \cong 0$, so the restriction of $g$ to $\Sigma \mathbb{C} P^{2}$ is null homotopic, implying that $g$ factors as a composite $\operatorname{Tel}\left(c_{i}\right) \longrightarrow \Sigma^{7} \mathbb{C} P^{2} \stackrel{h}{\longrightarrow} B G_{2}$ for some map $h$. By [16], $\pi_{9}\left(B G_{2}\right) \cong \mathbb{Z} / 2 \mathbb{Z}$. We claim that the restriction of $h$ to $S^{9}$ is trivial. If not, then it represents the generator $\gamma$ of $\pi_{9}\left(B G_{2}\right)$. By [16], this generator has the property that the composite $S^{10} \stackrel{\eta}{\longrightarrow} S^{9} \stackrel{\gamma}{\longrightarrow} B G_{2}$ is also nontrivial. But this implies that there can be no extension of $\gamma$ to a map $\Sigma^{7} \mathbb{C} P^{2} \longrightarrow B G_{2}$. That is, the restriction of $h$ to $S^{9}$ cannot extend 
to $h$, a contradiction. Therefore the restriction of $h$ to $S^{9}$ is trivial, implying that $h$ factors as a composite $\Sigma^{7} \mathbb{C} P^{2} \longrightarrow S^{11} \stackrel{k}{\longrightarrow} B G_{2}$ for some map $k$. By [16], $\pi_{11}\left(B G_{2}\right) \cong 0$. Hence $k$, and therefore $h$, and therefore $g$ are all trivial. Consequently, $\left[\operatorname{Tel}\left(c_{i}\right), B G_{2}\right] \cong 0$ for $2 \leq i \leq 3$. Collectively, we obtain a 2-local isomorphism $\left[G_{2} / T, G\right] \cong 0$.

Case 2: $p=3$. As in Section 5.3, there is a 3-local homotopy equivalence

$$
\Sigma G_{2} / T \simeq A(5,9) \vee \operatorname{Tel}\left(c_{2}\right) \vee \operatorname{Tel}\left(c_{3}\right) \vee \operatorname{Tel}\left(c_{4}\right) .
$$

By Lemma 6.1, to calculate the 3-component of $\left[G_{2} / T, G_{2}\right]$ it is equivalent to calculate $\left[A(5,9), B G_{2}\right]$ and $\left[\operatorname{Tel}\left(c_{i}\right), B G_{2}\right]$ for $2 \leq i \leq 4$. By [16], at 3 we have $\pi_{m}\left(B G_{2}\right)=0$ for $m \in\{3,5,9,11,13\}$ while $\pi_{7}\left(B G_{2}\right) \cong \mathbb{Z} / 3 \mathbb{Z}$. In particular, $\left[A(5,9), B G_{2}\right] \cong 0$ since $A(5,9)$ has cells in dimensions 5 and 9 , and $\left[\operatorname{Tel}\left(c_{3}\right), B G_{2}\right] \cong 0$ as $\operatorname{Tel}\left(c_{3}\right)$ has cells in dimensions 5,9 and 13.

The space $\operatorname{Tel}\left(c_{2}\right)$ has cells in dimensions 3,7 and 11 with the 7 and 11 cells connected by the Steenrod operation $\mathcal{P}^{1}$. The triviality of $\pi_{3}\left(B G_{2}\right)$ implies that any map $\operatorname{Tel}\left(c_{3}\right) \longrightarrow B G_{2}$ factors through $\operatorname{Tel}\left(c_{3}\right) / S^{3}$. The nontrivial Steenrod operation in cohomology implies that there is a homotopy cofibration

$$
S^{10} \stackrel{\alpha}{\longrightarrow} S^{7} \longrightarrow \mathrm{Tel}\left(c_{3}\right) / S^{3} \longrightarrow S^{11} .
$$

This induces an exact sequence

$$
\left[S^{11}, B G_{2}\right] \longrightarrow\left[\operatorname{Tel}\left(c_{3}\right) / S^{3}, B G_{2}\right] \longrightarrow\left[S^{7}, B G_{2}\right] \stackrel{\alpha^{*}}{\longrightarrow}\left[S^{10}, B G_{2}\right] .
$$

On the one hand, $\left[S^{11}, B G_{2}\right]=\pi_{11}\left(B G_{2}\right) \cong 0$. On the other hand, by $[16],\left[S^{7}, B G_{2}\right] \cong \mathbb{Z} / 3 \mathbb{Z}$, $\left[S^{10}, B G_{2}\right] \cong \mathbb{Z} / 3 \mathbb{Z}$, and $\alpha^{*}$ is an isomorphism. Thus $\left[\operatorname{Tel}\left(c_{3}\right) / S^{3}, B G_{2}\right] \cong 0$ and hence $\left[\operatorname{Tel}\left(c_{3}\right), B G_{2}\right] \cong 0$.

The space $\operatorname{Tel}\left(c_{4}\right)$ also has cells in dimensions 3, 7 and 11 , but this time there is no Steenrod operation connecting the 7 and 11 cells. Thus $\operatorname{Tel}\left(c_{4}\right) / S^{3} \simeq S^{7} \vee S^{11}$. As in the $\operatorname{Tel}\left(c_{2}\right)$ case, any map $\operatorname{Tel}\left(c_{4}\right) \longrightarrow B G_{2}$ factors through $\operatorname{Tel}\left(c_{4}\right) / S^{3} \simeq S^{7} \vee S^{11}$. The homotopy cofibration

$$
S^{3} \longrightarrow \operatorname{Tel}\left(c_{4}\right) \longrightarrow S^{7} \vee S^{11} \stackrel{\gamma}{\longrightarrow} S^{4}
$$

induces an exact sequence

$$
\left[S^{4}, B G_{2}\right] \stackrel{\gamma^{*}}{\longrightarrow}\left[\operatorname{Tel}\left(c_{4}\right), B G_{2}\right] \longrightarrow\left[S^{7} \vee S^{11}, B G_{2}\right] \longrightarrow\left[S^{3}, B G_{2}\right] .
$$

Since the 3 and 7-cells of $\operatorname{Tel}\left(c_{4}\right)$ are connected by the Steenrod operation $\mathcal{P}^{1}$, the restriction of $\gamma$ to $S^{7}$ is $\alpha$. It is not clear what the restriction of $\gamma$ to $S^{11}$ is but this is not relevant since $\pi_{11}\left(B G_{2}\right)=0$, so $\gamma^{*}$ factors as $\left[S^{4}, B G_{2}\right] \stackrel{\alpha^{*}}{\longrightarrow}\left[S^{7}, B G_{2}\right] \longrightarrow\left[S^{7} \vee S^{11}, B G_{2}\right]$. By $[16],\left[S^{4}, B G_{2}\right] \cong \mathbb{Z}$, $\left[S^{7}, B G_{2}\right] \cong \mathbb{Z} / 3 \mathbb{Z}$, and $\alpha^{*}$ is reduction mod-3. Therefore $\gamma^{*}$ is onto. On the other hand, $\left[S^{3}, B G^{2}\right] \cong 0$, so exactness in (5) implies that $\left[\operatorname{Tel}\left(c_{4}\right), B G_{2}\right] \cong 0$. Collectively, we obtain a 3-local isomorphism $\left[G_{2} / T, G_{2}\right] \cong 0$.

Case 3: $p=5$. As in Section 5.3, there is a 5-local homotopy equivalence

$$
\Sigma G_{2} / T \simeq 2 S^{5} \vee 2 S^{7} \vee 2 S^{9} \vee S^{13} \vee 2 A(3,11) \text {. }
$$

Therefore by Lemma 6.1

$$
\left[G_{2} / T, G_{2}\right] \cong 2 \pi_{4}\left(G_{2}\right) \times 2 \pi_{6}\left(G_{2}\right) \times 2 \pi_{8}\left(G_{2}\right) \times \pi_{12}\left(G_{2}\right) \times 2\left[A(3,11), B G_{2}\right] .
$$

By [16], $\pi_{4}\left(G_{2}\right) \cong 0$ and $\pi_{12}\left(G_{2}\right) \cong 0$, and localized at $5, \pi_{6}\left(G_{2}\right) \cong 0, \pi_{8}\left(G_{2}\right) \cong 0$. As well, $\pi_{3}\left(B G_{2}\right) \cong 0$ and $\pi_{11}\left(B G_{2}\right) \cong 0$ so $\left[A, B G_{2}\right] \cong 0$. Thus, at $5,\left[G_{2} / T, G_{2}\right] \cong 0$.

Case 4: $p>5$. As in Section 5.3, there is a $p$-local homotopy equivalence

$$
\Sigma G_{2} / T \simeq 2 S^{3} \vee 2 S^{5} \vee 2 S^{7} \vee 2 S^{9} \vee 2 S^{11} \vee S^{13} .
$$

By [16], the $p$-component of $\pi_{m}\left(G_{2}\right)$ is 0 for $m \in\{2,4,6,8,10,12\}$. Thus, at $p>5,[G / T, G] \cong$ 0 . 
Proposition 6.6. Localized away from 2 there is a group isomorphism $[S p(2) / T, S p(2)] \cong 0$.

Proof. The cells of $S p(2) / T$ occur in dimensions $2,4,6,8$, and by [17], after inverting 2 we have $\pi_{m}(S p(2)) \cong 0$ for $m \in\{2,4,6,8\}$. Thus $[S p(2) / T, S p(2)] \cong 0$.

\section{REFERENCES}

[1] F. Bergeron, N. Bergeron, R.B. Howlett, D.E. Taylor, A decomposition of the descent algebra of a finite Coxeter group, J. Algebraic Combin. 1 (1992) 23-44.

[2] I.N. Bernstein, I.M. Gelfand and S.I. Gelfand, Schubert cells and the cohomology of the spaces $G / P$, LMS 69, Cambridge Univ. Press (1982), 115-140.

[3] A. Borel, Sur la cohomologie des espaces fibrés principaux et des espaces homogènes de groupes de Lie compacts, Ann. of Math. 57 (1953), 115-207.

[4] A. Borel, Sous-groupes commutatifs et torsion des groupes de Lie compacts connexes, Tôhoku Math. J. (2) 13 (1961), 216-240.

[5] R. Bott and H. Samelson, The integral cohomology ring of G/T, Proc. Nat. Acad. Sci. USA 41 (1955), 490-493.

[6] H. Duan and X.A. Zhao, The classification of cohomology endomorphisms of certain flag manifolds, Pacific J. Math. 192 (2000), 93-102.

[7] H. Duan and X.A. Zhao, A unified formula for Steenrod operations in flag manifolds, Compositio Mathematica, 143 (2007), 257-270.

[8] C. Chevalley, Sur les décomposition cellulaires des espaces $G / B$, Algebraic Groups and their Generalizations: Classical Methods (W. Haboush, ed.), Proc. Sympos. Pure Math., 56, Part 1, Amer. Math. Soc., 1994, 1-23.

[9] H. Glover and W. Homer, Self-maps of flag manifolds, Trans. Amer. Math. Soc. 267 (1981), 423-434.

[10] P. Hilton, G. Mislin, and J. Roitberg, Localization of nilpotent groups and spaces, Math. Studies No. 15, North-Holland, Amsterdam, 1975.

[11] S. Kaji, Three presentations of torus equivariant cohomology of flag manifolds, to appear in Proceedings of International Mathematics Conference in honour of the 70th Birthday of Professor S. A. Ilori, arXiv.org/1504.01091.

[12] N. Kitchloo, Cohomology splittings of Stiefel manifolds, J. London Math. Soc. (2) 64 (2001), no. 2, 457-471.

[13] A. Lascoux and M. Schützenberger, Polynômes de Schubert, C. R. Acad. Sci. Paris Sér. I Math. 294 (1982), no. 13, 447-450.

[14] J. P. May and K. Ponto, More concise algebraic topology, Chicago Lectures in Mathematics. University of Chicago Press, Chicago, IL, 2012.

[15] H. Miller, Stable splitting of Stiefel manifolds, Topology, 24(4) (1985), pp. 411-419.

[16] M. Mimura, The homotopy groups of Lie groups of low rank, J. Math. Kyoto Univ. 6 (1967), 131-176.

[17] M. Mimura and H. Toda, Homotopy groups of $S U(3), S U(4)$ and $S p(2)$, J. Math. Kyoto Univ. 3 (1964), 217-250.

[18] G. Nishida and Y. Yang, On a p-local stable splitting of $U(n)$, J. Math. Kyoto Univ. 41 (2001), no. 2, 387-401.

[19] S. Papadima, Rigidity properties of compact Lie groups modulo maximal tori, Math. Ann. 275 (1986), 637-652.

[20] S. Priddy, Recent progress on stable splittings, Proc. Durham Symp. on Homotopy Theory 1985, LMS 117 (1987), 149-174, Cambridge Univ. Press.

[21] J.R. Stembridge, Orthogonal sets of Young symmetrizers, Adv. in Appl. Math. 46 (2011), 576-582.

[22] H. Toda, Composition methods in homotopy groups of spheres, Annals of Mathematics Studies 49, Princeton University Press, 1962.

[23] $\mathrm{H}$. Toda and T. Watanabe, The integral cohomology ring of $F_{4} / T$ and $E_{6} / T$, J. Math. Kyoto Univ. 14 (1974), 257-286.

[24] H. E. Ullman, An equivariant generalization of the Miller splitting theorem, Algebr. Geom. Topol. 12 (2012), no. 2, 643-684.

[25] Y. Yang, On a p-local stable splitting of Stiefel manifolds, J. Math. Soc. Japan 54 (2002), 911-921.

[26] X.A. Zhao, Cohomology endomorphisms of flag manifolds, Acta Math. Sinica 44 (2001), 1099-1106.

[27] X.A. Zhao, Maps from a simply connected space to a flag manifold G/T, Acta. Math. Sinica 20 (2004), 1131-1134. 
Institute of Mathematics for Industry, Kyushu University, Fukuoka, 819-0395, Japan.

E-mail address: skaji@imi.kyushu-u.ac.jp

Mathematical Sciences, University of Southampton, Southampton SO17 1BJ, United Kingdom

E-mail address: s.d.theriault@soton.ac.uk 